\title{
Homeobox B7 is a Newly Hypothesized Oncogene Promoting Tumorigenesis in Head and Neck Squamous Cell Carcinoma
}

\section{Xiang Wu}

Nanjing Medical University

Jin Li

Nanjing Medical University

Tingyuan Yan

Nanjing Medical University

\section{Xueping Ke}

Nanjing Medical University

\section{Xin Li}

Nanjing Medical University

\section{Yumin Zhu}

Nanjing Medical University

Jianrong Yang

Nanjing Medical University

Zhongwu Li ( $\sim$ lizhongwu@njmu.edu.cn )

Nanjing Medical University https://orcid.org/0000-0002-8378-3535

\section{Primary research}

Keywords: head and neck squamous cell carcinoma, HOXB7, stemness, EMT, prognostic markers

Posted Date: April 12th, 2021

DOI: https://doi.org/10.21203/rs.3.rs-351812/v1

License: (c) (1) This work is licensed under a Creative Commons Attribution 4.0 International License. Read Full License

Version of Record: A version of this preprint was published at Cancer Cell International on July 24th, 2021. See the published version at https://doi.org/10.1186/s12935-021-02093-6. 


\section{Abstract}

Background: The core member of Homeobox gene family, Homeobox B7 (HOXB7), deregulating in multiple cancers, is considered as a pro-tumorigenic transcription factor. However, the pro-tumorigenic role in head neck squamous cell carcinoma (HNSCC) have not been reported yet.

Methods: Publicly available databases of HNSCC were used to datamining the expression of HOXB7 in HNSCC. Immunohistochemistry was used to measure protein level in a retrospective cohort of primary HNSCC specimens with assessment of the associations between $\mathrm{IHC}$ results and various clinicopathological parameters and patient outcome data. The pro-tumorigenic roles of HOXB7 in HNSCC were further delineated in vitro and in vivo by loss-of-function assay. Cancerous analysis \& treatment strategy of HOXB7 was assessed by combination of functional enrichment analysis and the Connectivity Map (CMap) analysis.

Results: In 8 HNSCC cohorts, HOXB7 mRNA was significantly over-expansion. HOXB7 protein was markedly upregulated in HNSCC samples as compared to normal counterparts and its overexpression significantly associated with large pathological grade, clinical stage, cervical node metastasis (chi-square test, $P=.03, .0152, .0195)$ and reduced overall and disease-free survival (Kaplan-Mier analyses, Log-rank test, $P=.0007, .0014)$. Univariate and multivariate survival analyses further revealed HOXB7 protein abundance as an independent prognostic factor for patients' overall survival. Moreover, HOXB7 knockdown significantly inhibited cell proliferation, migration and invasion, stemness maintenance, and induced cell apoptosis in HNSCC cells, and resulted in compromised tumour growth in vivo. Furthermore, we screened 3 small molecule drugs potentially effective targeting HOXB7 by performing a CMAP analysis.

Conclusions: This research on HOXB7 developed a framework to identify a robust novel cancer biomarker on diagnosis and prognosis, and given a potentially available therapeutic strategy for the treatment of HNSCC.

\section{Introduction}

Head and neck squamous cell carcinoma (HNSCC) is a type of solid malignancy initiated form squamous epithelium and originated from oral cavity, larynx, and pharynx, the incidence of who ranks sixth in malignancy worldwide [1]. Smoking, drinking, chewing areca nut, and human papillomavirus infection are considered to be the most important risk factors for HNSCC. Currently, HNSCC is treated with a combination of surgical resection, radiotherapy, and chemotherapy, but the 5-year survival rate is not significantly improved $[2,3]$. However, the validity and specificity of the traditional predictive parameters such as clinical stage, depth of invasion, surgical margin and involvement of cervical lymph nodes are relatively low and cannot meet the clinical needs $[2,3,4]$. Therefore, it is important to find more accurate and reliable biomarkers for the early diagnosis, prognosis prediction and guidance for therapy selection 
for patients who suffering from HNSCC, while, also contribute to the development of molecular targeted therapies for this deadly disease.

Homeobox (Hox) family members are the indispensable transcription factors in mammal embryonic development which regulate cell differentiation and morphogenesis. Every family member protein contains a consensus sequence - homeodomain, which encodes by a 61 -amino-acids signature $[5,6]$. The mRNA expression of Hox family members has tumor heterogeneity, but often detected aberrantly upgradation, which may lead to transcription of tumor associated genes [6]. In this research, we focus on Homeobox B7 (HOXB7) which is located on the 17q21.3 human chromosome and has been reported to be aberrantly expressed in various malignancies, such as oral cancer [7], lung cancer [8], breast cancer [9], gastric cancer [10], liver cancer [11] and esophageal cancer [12]. While, the excessive overexpression of HOXB7 has highly confidence correlated with disease advancement and poor outcome, which serve as a potential therapeutic biomarker $[13,14]$. HOXB7 is responsible for many of the major cellular processes that occur in cancer, including proliferation, invasion, migration, angiogenesis and the epithelialmesenchymal transition (EMT) $[12,13,14,15]$. Although the biological functions of HOXB7 in various cancers have been described, its function in the controlling tumorigenesis and tumor progression of HNSCC has not been well characterized.

In this study, we confirmed that HOXB7 is upregulated in the clinical specimens, TCGA and GEO databases, which is associated with poor outcomes in HNSCC patients. Additionally, we investigated the biological function of the HOXB7 protein in HNSCC cell lines. Our results suggest that HOXB7 knockdown inhibits HNSCC metastasis by decreasing EMT-related markers in vitro. Furthermore, knowdown of HOXB7 reduces HNSCC tumor spheroidizing ability by decreasing expression of stem cell-related markers in vitro. Surprisingly, HOXB7 had strongly effect on HNSCC proliferation in vitro and in vivo and induced cell apoptosis in vitro. In summary, our study highlights the prognostic value of HOXB7 in HNSCC. To the best of our knowledge, this is the first report to indicate the role of HOXB7 in primary tumor samples, TCGA and GEO datasets from HNSCC patients and to investigate the probable mechanism by which HOXB7 contributes to HNSCC cell migration, invasion and proliferation etc by bioinformatics analysis.

\section{Materials And Methods}

\section{HNSCC samples}

A total number of 119 tissue samples of patients with primary HNSCC were collected from patients who underwent curative resection (January 2012 - September 2015) at the department of oral and maxillofacial surgery, Affiliated Stomatological Hospital of Nanjing Medical University, None of the patients had received adjuvant chemotherapy, radiation or any other treatment before resection, and all patients have detailed demographic, clinical, pathological and follow-up data. 26 normal adjacent oral mucosa samples obtained from donors during non-tumor surgeries were also included. This study was performed in accordance with guidelines outlined in the 1964 declaration of Helsinki and was approved by the Ethic Committee of Nanjing Medical University. 


\section{Cell culture}

Five HNSCC cell lines including SCC9, SCC25, Cal27, FaDu and a normal human oral keratinocytes (HOK) were obtained from American Type Culture Collection (ATCC,USA). And HN4 and HN6 were generously gifted from Dr. Wantao Chen (Shanghai Jiao Tong University). HNSCC cells were cultured in DMEM/F12 (Invitrogen, USA) with 10\% fetal bovine serum (FBS, Gbico, USA) in a humidified atmosphere with $5 \%$ CO2 at $37^{\circ} \mathrm{C}$

\section{Small interfere RNAs, lentivirus production and cell transfection}

The siRNAs against HOXB7 with siNC as a negative control were obtained from GenePharma (Shanghai, China). The and sequences of the HOXB7 siRNA including siRNA-1: 5'-GCUAUUGUAAGGUCUUUGUTT, 5'ACAAAGACCUUACAAUAGCTT; siRNA-2: 5'-CCCUUUGAGCAGAACCUCUTT, 5'-

AGAGGUUCUGCUCAAAGGGTT; The final concentration of $100 \mathrm{nM}$ siRNA or siNC pre-coated with Lipofectamine 3000 (Invitrogen, USA) were used for transfection.

Lentiviral vectors encoding the short hairpin RNAs (shRNAs) that target HOXB7 with the sequence of and a scramble shRNA were purchased from GenePharma (Shanghai, China). Transfection processes were conducted according to the instructions provided by the manufacturer. To generate the stable cell line, The transduced cells were then selected in culture medium containing puromycin $(5.0 \mu \mathrm{g} / \mathrm{ml})$.

\section{RNA extraction and quantitative real-time PCR}

Total RNA was extracted by trizol reagent according to the manufacturer's protocol. Two micrograms of RNA was reverse-transcribed into cDNAs and subjected to PCR reactions using the Prime-ScriptTM RTPCR kit (Takara). Primers used for real-time PCR were as follows: HOXB7, forward: 5'TTCCCAGAACAAACTTCTTGTGC-3'; reverse: 5'-GCATGTTGAAGGAACTCGGCT-3'. 18sRNA, forward: 5'ACACGGACAGGATTGACAGA-3'; reverse: 5'-GGACATCTAAGGGCATCACA-3'. All of the determinations were performed in duplicate. The relative expression of HOXB7 mRNA was normalized to the expression level of 18 sRNA mRNA using the $2-\Delta \mathrm{Ct}$ method.

\section{Western blot analysis}

Total protein was extracted from tumor cells. Equal amounts of protein were loaded onto a 10\% SDSPAGE and electrophoresed and transferred onto PVDF membrane for 60-90 min based on the molecular weight of the target protein. After the membranes were blocked with $5 \%$ non-fat milk, they were incubated overnight at $4{ }^{\circ} \mathrm{C}$ with the following primary antibodies: HOXB7 (1:1000,H00003217-M03, Abnova), vimentin (1:2000, \#5741, Cell signaling, USA), E-cadherin (1:2000, \#3195, Cell signaling), N-cadherin (1:1000, \#13116, Cell signaling), CD44(1:1000, \#6024-1-Ig, Proteintech, USA), CD133(1:1000, \#18470-1AP, proteintech), ALDH1A1(1:1000, \#15910-1-AP, proteintech), Bmi1 (1:1000, Bmi1, Cell signaling), SOX2(1:1000, \#4900, Cell signaling) and GAPDH (1:5000, MB001, Bioworld, China) followed by incubation with horseradish peroxidase(HRP)-conjugated secondary antibodies. Immunoreactive bands on the blots were detected by ECL chemiluminescence kit (Bio-Rad, USA). 


\section{CCK-8 and BrdU assay}

Cell Counting Kit-8 (CCK8) assay (Cell Counting Kit-8, Dojindo, Japan) was used to measure cell viability. After transfection, cells were placed in 96 -well plates at a density of $2 \times 10^{3}$ cells per well; the absorbance values were detected 0 to 3 days after transfection. $10 \mu \mathrm{L}$ of CCK-8 solution was added daily to each well flled in the 96-well plates and incubated for another $2 \mathrm{~h}$. Then, a microplate reader (Multiskan MK3, Thermo, USA) was used to measure the absorbance at $450 \mathrm{~nm}$. For BrdU assay, $2 \times 10^{5}$ cells were inoculated into a 6-well culture plate (with a cover slip placed inside) for $24 \mathrm{~h}$, then incubated with 1.0 $\mathrm{mg} / \mathrm{mL}$ BrdU solution (Applied Biosystems, USA) for $4 \mathrm{~h}$. The culture solution was then discarded, followed by cell fixation in methanol for $10 \mathrm{~min}$ and cell staining in diamidine phenyl indoles (DAPI; Thermo Fisher Scientific). BrdU-positive cells were arbitrarily counted in three visual fields through the microscope.

\section{Tumorsphere formation assay}

In total, $2 \times 10^{4} / \mathrm{ml}$ cells were plated onto ultra-low-attachment plates (NUNC, Thermo, USA) and cultured in DMEM/F12 serum-free medium (Invitrogen, USA) supplemented with B27 (BD Bioscience), $10 \mathrm{ng} / \mathrm{mL}$ bfibroblast growth factor (bFGF, BD Bioscience, USA), $20 \mathrm{ng} / \mathrm{mL}$ epidermal growth factor insulin (BD Bioscience). The number of spheres was captured and counted under an inverted microscope after 10 days.

\section{Cell apoptosis assessed by flow-cytometric assay}

Cells were harvested and resuspended in $500 \mu$ l of binding buffer, and stained with Annexin V-FITC/PI Apoptosis kit (BD Biosciences). Apoptosis percentages were then detected using a FACSCaliber flow cytometer (BD Biosciences) and analyzed by Flowjo V10.1.

\section{In vitro cell invasion and wound healing assay}

Cell invasion were determined by a Matrigel transwell invasion assay. Cells $(1 \times 105 /$ well $)$ were suspended in $200 \mu \mathrm{l}$ of serum-free DMEM and added to the upper chamber of an insert (8 $\mu \mathrm{m}$ pore size, Millipore, Germany) coated with Matrigel (BD Biosciences, USA). And Then, $600 \mu$ DMEM with $10 \%$ FBS was added to the lower chamber. For wound healing assay, Cells $\left(2 \times 10^{5}\right.$ cells/well.) were seeded onto a 6 -well plate overnight. The confluent monolayers were scratched using sterile pipette tips and washed with phosphatebuffered saline (PBS) 3 times to remove detached cells. The wounds were photographed at 0 , 6,12 and $24 \mathrm{~h}$ as indicated.

\section{Immunofluorescence assay}

Cells were seeded on glass coverslips $18 \mathrm{~h}$ prior to experiment and fixed with $4 \%$ paraformaldehyde and washed thoroughly with PBS. then permeabilized in 0.1\% Triton X-100 (Sigma-Aldrich). The cells were washed with PBS and blocked with $3 \%$ bovine serum albumin (BSA) for $30 \mathrm{~min}$ at $37^{\circ} \mathrm{C}$. Then, incubation with primary antibodies against HOXB7 overnight. Cell were followed by incubation with secondary 
antibodies for $1 \mathrm{~h}$. DAPI was used to counterstain DNA. Immunofluorescence images for HOXB7 were viewed with Zeiss fluorescence microscope.

\section{Immunohistochemical staining and scoring}

Paraffin-embedded tissue samples from HNSCC patients were sliced into 4- $\mu$ m-thick sections. Tumor

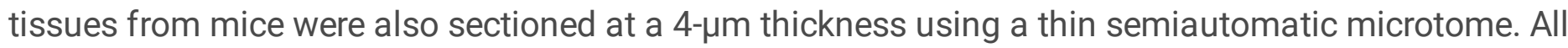
sections were deparaffinized in xylene and rehydrated in a series of graded alcohol dilutions. Antigen retrieval was performed by heating in a microwave oven. Then, the sections were incubated with $3 \%$ $\mathrm{H} 2 \mathrm{O} 2$ for 10 min followed by $10 \%$ normal goat serum for $15 \mathrm{~min}$ at room temperature to block endogenous peroxidases and non-specific antigens. Histological sections were immunostained overnight at $4{ }^{\circ} \mathrm{C}$ using the following primary antibodies: anti-HOXB7 antibody (1:200, \#H00003217-M03, Abnova), anti-CD133 antibody, anti-CD44 antibody and anti-Ki67 antibody. Negative controls (only PBS incubation) were included in each staining run. Immunoreactivity in each slide was semi-quantitatively evaluated according to staining intensity and distribution and the immunoreactive score was calculated as intensity score $\times$ proportion score. Intensity score was defined as 0 , negative; 1 , weak; 2 , moderate; 3 , strong, while the proportion score was evaluated by two independent pathologists via counting positive nucleus with 0 , negative; $1,<10 \% ; 2,11-50 \% ; 3,51-80 \% ; 4,>80 \%$ positive cells. The immunoreactivity of each slide was categorized into three subgroups according to the final score: 0 , negative; $1-4$, low expression; $\geq 4$, high expression.

\section{HNSCC xenograft animal model}

All experiments involving animal subjects were in accordance with the institutional animal welfare guidelines and approved by Institutional Animal Care and Use Committee of Nanjing Medical University. Six-week-old female NOD/SCID mice were purchased from Model Animal Research Center of Nanjing Medical University and maintained in the specific pathologic-free animal facility. $2 \times 10^{6}$ cells of FaDu in $100 \mu \mathrm{L}$ PBS then subcutaneously injected into both flanks of each animal ( 6 animals per experimental group). Sizes of tumors and were measured every 3 days when tumour masses were identified. Tumor volume $=[($ length $) \times($ width $) \times($ width $)] / 2$. All the mice involved was sacrificed by intraperitoneal injection of a deadly dose of pentobarbital sodium $(150 \mathrm{mg} / \mathrm{kg})$ at the $31^{\text {th }}$ day after tumor cell injection. When the vital signs of mice disappeared, the end-point tumor was dissected, weighed and recorded.

\section{Bioinformatics analysis of HOXB7 from public databasesData Sources}

The RNA sequencing and clinical data were obtained from The Cancer Genome Atlas (TCGA) database. The microarray datasets: GSE6631, GSE12452, GSE23036, GSE25099, GSE30784, GSE42743 and GSE9844 were downloaded from the Gene expression Omnibus database.

\section{Functional enrichment of HOXB7 co-expression genes}


In this study, we used the Pearson correlation coefficient $(r)$ to screen and identify HOXB7-related genes with a $P<0.05$ and $|r|>0.3$ were identified as HOXB7-related genes. The biological functions of these HOXB7 co-expression genes were comprehensively detected by GO enrichment and kyoto encyclopedia of genes and genomes (KEGG) pathway analysis. In addition, the protein-protein interactions (PPIs) among all HOXB7 co-expression genes were obtained by Search Tool for the Retrieval of Interacting Genes (STRING; http://string-db.org) and the network was constructed with the Cytoscape 3.7.0 software.

\section{Survival analysis and small molecule targeted drugs screening of HOXB7 in HNSCC}

The Connectivity Map (CMap) (http://www.broad.mit.edu/cmap)was used to identify potential small molecule targeted drugs for HOXB7 in HNSCC. Those small molecule drugs with mean connective score $<-0.2$ and $\mathrm{P}<0.05$ were recognized as the possible therapeutic drugs of HOXB7 in HNSCC.

\section{Statistical analysis}

Data between two groups were examined using a two-tailed paired Student's t-test or or ANOVA (Bonferroni post hoc test). The Chi-squared test was applied to assess the correlation between HOXB7 expression and various clinicopathological parameters. Survival data were used to establish KaplanMeier curves, and the differences among the groups were analyzed by the log rank test. Univariate and multivariate Cox's proportional regression analysis were employed to determine prognostic factors associated with survival. Two-tailed $P$ values $<0.05$ were considered as statistically significance. All statistical analyses were performed by GraphPad Prism 7 or R (4.0.2).

\section{Results}

\section{Carcinogenic analysis of HOXB7 in HNSCC from public datasets}

Mounting cancer research as well as pan-cancer analysis have revealed that overexpressed HOXB7 has to do with unfavorable outcomes $[7,10,13,16]$. Firstly, we used the publicly available sets such as TCGA and GEO datasets to analysis the relevant information on HOXB7 expression patterns. Integration and analysis of the TCGA-HNSC cohort (502 cases) data showed that HOXB7 mRNA was obviously upregulated in the TCGA-HNSC specimens compared with the normal counterpart (44 cases) using TCGA dataset (Fig. 1A). While as displayed in Fig. 1B-H, data mining and questioning from GSE6631, GSE12452, GSE23036, GSE25099, GSE30784, GSE42743 and GSE9844 indicated that HOXB7 mRNA was significantly higher in HNSCC cancerous samples, respectively. Multiple evidence has indicated that cancer patients accompany with aberrantly overexpression of HOXB7 have more risk ending with adverse outcome $[10,13,14,19]$.

\section{Hoxb7 Is Highly Upregulation In Hnscc}


To further find out the expression and enrichment pattern of HOXB7 in HNSCC specimens, we next performed immunohistochemical staining of HOXB7 from our clinical cohort which contains 119 primary HNSCC samples. As displayed in Fig. 2A-F, HOXB7 showed a positive staining mainly in nucleus in cancerous paraffin sections, whereas weak/negative staining was identified in the normal mucosa and the stroma of HNSCC. Based on our IHC-scoring system, HOXB7 expression in HNSCC/normal mucosa was classified, in HNSCC (high, $n=71$ versus low, $n=48$ ) and in normal clinical specimens (high, $n=1$ versus low, $n=4$ versus negative, $n=8$ ). Therefore, these data confirmed that the HOXB7 protein was highly expressed in HNSCC $(P<0.001$, Fisher's exact test. Then, detailed clinical data of this cohort is given in Table 1 (52 females versus 67males, average 61.49 years). The patient's latest follow-up time ranged from 3 to 83 months). The correlation between HOXB7 expression and clinicopathological parameters were displayed in Table 1 (Chi-square test). It is clear that there were no obviously association between HOXB7 abundance and patients age, drinking and heavy tobacco usage, tumor size and gender. Notably, HOXB7 expression was positively associated with cervical lymph nodes metastasis, pathological grade and clinical stage with $P$ value $0.0195,0.03$ and 0.0152 (Chi-square test), respectively. When we have known the HOXB7 mRNA abnormal expansion in HNSCC tissues, next use HNSCC cell lines to validated. Firstly, we detected HOXB7 level form mRNA and protein aspects in a panel of HNSCC cell lines (versus with the normal oral keratinocytes HOK). As shown in Fig. 5A-B, HOXB7 transcriptional levels in all cancerous cells were significantly higher than that in HOK as assessed by qRT-PCR assay. Moreover, immunofluorescence was performed to visualized the subcellular distribution of HOXB7 protein in both FaDu and Cal27 cells. As data showed in Fig. 2G-H, HOXB7 was mainly enriched in nucleus but much less in cytoplasm in both cell lines which consistent with the TFs' location. 
Table 1

The associations between HOXB7 expression and multiple clinicopathological parameters in HNSCC Samples

\begin{tabular}{|c|c|c|c|c|}
\hline \multirow[t]{2}{*}{ Clinicopathological parameters } & \multirow[t]{2}{*}{ Samples } & \multicolumn{2}{|c|}{ HOXB7 } & \multirow[t]{2}{*}{$P$ values } \\
\hline & & High & Low & \\
\hline Gender & & 71 & 48 & 0.4455 \\
\hline Male & 67 & 42 & 25 & \\
\hline Female & 52 & 29 & 23 & \\
\hline Age & & & & 0.2631 \\
\hline$\leq 60$ & 57 & 37 & 20 & \\
\hline$>60$ & 62 & 34 & 28 & \\
\hline Smoking & & & & 0.3508 \\
\hline No & 81 & 46 & 35 & \\
\hline Yes & 38 & 25 & 13 & \\
\hline Alcohol & & & & 0.2404 \\
\hline No & 90 & 51 & 39 & \\
\hline Yes & 29 & 20 & 9 & \\
\hline Pathological grade & & & & 0.03 \\
\hline I & 65 & 33 & 32 & \\
\hline$\| I-I I$ & 54 & 38 & 16 & \\
\hline Clinical stage & & & & 0.0152 \\
\hline$|-| \mid$ & 51 & 24 & 27 & \\
\hline III-IV & 68 & 47 & 21 & \\
\hline Tumor size & & & & 0.3508 \\
\hline T1-T2 & 81 & 46 & 35 & \\
\hline T3-T4 & 38 & 25 & 13 & \\
\hline Cervical node metastasis & & & & 0.0195 \\
\hline$N(0)$ & 69 & 35 & 34 & \\
\hline$N(+)$ & 50 & 36 & 14 & \\
\hline
\end{tabular}


HOXB7 aberrant overexpression associates with reduced survival in HNSCC patients

To explore relationship between HOXB7 abundance and prognosis of patients with HNSCC, we attempted to evaluate the relationship between HOXB7 protein expression and clinical outcomes. According to the last follow-up data, 57 (47.9\%) patients were still disease-freely alive, $11(9.2 \%)$ survival with cervical nodal metastasis and/or local recurrences, whereas 51 (42.9\%) patients died of post-surgical relapse, cancer metastases or other diseases. Furthermore, through Kaplan-Meier analysis, patients with high HOXB7 abundance had obviously shorter overall-survival and disease-free survival than patients with low (Log-rank, $P=0.0007,0.0014$, Fig. 3A, B). Whereas, the similar conclusion form TCGA-HNSC cohort showed that the overall survival proportions in HOXB7 high groups was also significantly lower than those in HOXB7 low expression groups (Log-rank, $P=0.032$, Fig. 3C), however, in disease-free survival, no significantly result was observed (Log-rank, $P=0.08$, Fig. 3D).

To go a step further, univariate and multivariable survival analyses were executed by cox proportional hazards regression model. In the univariate analysis, pathological grade [hazard ratio (HR), 2.656; 95\% confidence interval $(95 \% \mathrm{Cl}), 1.491-4.729 ; P=0.0009]$, cervical nodal metastasis[HR, $2.453 ; 95 \% \mathrm{Cl} 1.39-$ 4.329; $P=0.0019]$, clinical stage [HR, 2.493; 95\% Cl, 1.304-4.766; $P=0.0058]$ and HOXB7 expression [HR, $2.875 ; 95 \% \mathrm{Cl}, 1.529-5.404 ; P=0.0010]$ significantly associated with overall survival, while other clinicopathological variable did not reach the statistical significance as indicated in Fig. 4A. To eliminate the confounding factor, multivariate survival analysis included gender, tumor size, pathological grade, pathological grade and HOXB7 expression. In this cox regression model, HOXB7 abundance was found to be an independent prognostic marker for the overall survival of patients with HNSCC (HR, 2.2481; $95 \% \mathrm{Cl}$, $1.1230-4.5004 ; P=0.02216)$, together with pathological grade $(\mathrm{HR}, 2.432 ; 95 \% \mathrm{Cl}, 1.2578-4.7022 ; \mathrm{P}=$ 0.00820 ) (also shown in Fig. 4B).

\section{HOXB7 depletion impairs proliferation and triggers apoptosis in HNSCC cells in vitro}

Recent discoveries and rapidly accumulating data have revealed that HOXB7 is a potential protumorigenic role driving tumorigenesis [20,21,22]. However, its oncogenic roles in HNSCC initiation and progression still uncovered yet. To characterize the biological roles of the HOXB7 in HNSCC, we first carried out the loss-of-function assay by siRNA-mediated loss of function approach. As displayed in Fig. 5C-D, Cal27 and FaDu cells with relatively higher expression of endogenous HOXB7 protein were shortlisted for further loss-of-function assays. Two independent siRNAs targeting human-HOXB7 (siHOXB7-1, siHOXB7-2) were introduced into FaDu and cal27 cells to detect the changes of HOXB7 protein and mRNA expression and changes of cell phenotypes. As shown in Fig. 5C-D, the expression of HOXB7 protein and mRNA decreased significantly after transfection with siHOXB7, which confirmed the effectiveness of our loss-of-function assay. And then, we detected the phenotypic changes associated with HOXB7 knockdown. Following HOXB7 downregulated, the proliferation and viability of both FaDu and Cal27 cells were obviously impaired as measured by BrdU (Fig. 5G,H,J,K) and CCK-8 assays (Fig. 5EF). The results showed that the ability of cell proliferation and survival decreased significantly in siHOXB7-treated cells. Moreover, Annexin V-PI Flow cytometric experiment showed a obvious increased in 
apoptosis rate of siHOXB7-treated cells. The apoptosis ratios were increased from $5.2-22.6 \%$ in Cal27 and from $3.8-13.7 \%$ in FaDu, respectively (Fig. 5I, L, M).

\section{HOXB7 knockdown inhibited migration and invasion and was involved in CSC maintenance in HNSCC}

To futher determine whether HOXB7 participates in the regulation process of EMT and CSC maintenance in HNSCC, wound healing and transwell experiments were executed to examine the migration and invasion ability of HOXB7 knockdown cells, respectively. siHOXB7 treated cells obviously diminished the ability of cell migration (Fig. 6B) and invasion (Fig. 6C, D, E) in vitro. Consistent with these observed phenotypical varies following HOXB7 knockdown, the protein expression of EMT/metastasis-associated markers Snail[Vimentin and N-cadherin were decreased and accompanied by adhere markers E-cadherin increased-expression (Fig. 6A). And then, tumorsphere formation assay performed and displayed in Fig. 5G, H, I, comparing with siNC group, tumorsphere formatting ability of siHOXB7-treated cells was pronouncedly impair in Cal27 and FaDu cells. Moreover, the abundance of related CSC markers (ALDH1A1, CD44, CD133, Bmi-1, and SOX2) were obviously reduced upon HOXB7 inhibition (Fig. 6F). All of the above results reveal that HOXB7 is participated in the regulation of malignant phenotypes of HNSCC, suggesting that it may be a very valuable potential therapeutic target.

\section{HOXB7 knockdown impairs tumour growth in an HNSCC xenograft model}

To further confirm the carcinogenicity of HOXB7 in vivo, we established an HNSCC xenograft model by inoculating FaDu cells stably depleted of HOXB7 into the left abdomen of nude mice and then monitoring the occurrence and growth of tumors after cell injection. As shown in Fig. 7A-C, compared with control cells, tumor growth in xenograft samples formed by siHOXB7-treated cells was impaired, and tumor volume and weight were significantly reduced. However, two weeks after cell transplantation, the incidence of tumor masses formed in HOXB7 damaged cells and control cells was comparable. Immunohistochemical staining of tumor samples showed that the positive staining of stem cell markers in HOXB7 knockdown cell samples was significantly reduced compared with the control (Fig. 7D). In addition, the number of Ki67-positive cells in tumor samples derived from HOXB7 knockdown cells was significantly reduced compared to samples formed from control cells (Fig. 7D). Together, these findings indicate that HOXB7 knockdown impairs HNSCC tumor growth in vivo, suggesting that HOXB7 may be required for HNSCC growth.

\section{Therapeutic Potential Analysis On Hoxb7 In Hnscc}

In order to investigate HOXB7 therapeutic potentiality, firstly, we conducted a genome-wide co-expression analysis of HOXB7 to explore pro-tumorigenic functions, and screened $198 \mathrm{HOXB7}$ related genes and all genes were positively correlated with HOXB7 (Fig. 8A). Then we carried out GO analysis for these coexpressed genes and these HOXB7 related genes were participated in Organelle fission, Nuclear division, DNA replication, Chromosome region, ATPase activity and catalytic activity, Acting on DNA (Fig. 8B). 
KEGG analysis showed that HOXB7 related genes enriched in multiple pathways such as Cell cycle, DNA replication and Homologous recombination (Fig. 8C).

To further explore HOXB7 as a potential therapeutic target for HNSCC, we performed a CMAP analysis with the screening conditions under mean connective score $<-0.2$ and $P<0.05$, and finally screened 3 small molecule drugs potentially effective targeting HOXB7. They are NU-1025 (Mean connective score $=$ -0.560; $P=0.003$ ), thiamine (Mean connective score $=-0.550 ; P=0.038$ ), vinburnine (Mean connective score $=-0.410 ; P=0.018$, and the chemical structures of which are shown in Supplementary Fig. 1 . Those small molecule drugs with were recognized as the possible therapeutic drugs of HOXB7 in HNSCC.

\section{Discussion}

Nowadays, it is still an urgent mission to find new biomarkers to provide new targets for the treatment of HNSCC especially under the condition of long-term survival rate of HNSCC patients has not improved significantly $[23,24]$. HOXB7, a typical transcription regulator, encodes a homologous protein which is not only related to the normal development and differentiation of cells or organs, but also abnormal highly expressed in tumor cells or tissues, and participates in tumor initiated and progression. Up to now, a great quantity studies have revealed that the abnormal expression of HOXB7 was closely associated with the malignant changes and poor prognosis of human tumors $[10,11,12,13,14,15,16,17]$. Here, combined with bioinformatics and clinical samples analysis, we found that HOXB7 protein and mRNA were significantly overexpressed in HNSCC, the highly expression of whom is closely related to clinical staging, lymph node metastasis and high risk of poor prognosis in patients. In addition, HOXB7 knock-down significantly impaired cell proliferation, migration and invasion and induced apoptosis in vitro and vivo. Moreover, HOXB7 RNAi-related silencing significantly inhibited invasion and migration, and reduced the ability of tumor spheres formation in vitro. Bioinformatics analysis supports that HOXB7 participates in the regulation of tumor malignant phenotype and can be used as a therapeutic target for small molecule compounds. Our results together others strongly suggested that as a newly hypothesized oncogene HOXB7 promotes HNSCC development and also a novel biomarker with clinical translation potential.

It is undisputed that dysregulated of HOXB7 in multiple types of cancers associated with cancer progression $[13,14,15,16,17,18,19]$. Here, we found that HOXB7 was abnormally overexpression in most HNSCC samples as confirmed via HOXB7 mRNA uncommonly up-regulated in various cancer clinical data sets and HOXB7 protein overexpression in our HNSCC cohort. Previous studies have shown that abnormally elevated HOXB7 expression is significantly associated with tumor size, cervical lymph node metastasis, malignancy, and etc. $[15,16,17,19]$. After establishing the overexpression pattern of HOXB7 in HNSCC, we then found that its abnormal expression is closely related to cervical lymph node metastasis and clinical staging, while did not reach statistical significance with other clinicopathological parameters. Moreover, Kaplan-Meier survival analysis showed HNSCC patients with higher HOXB7 expression has poorer prognosis risk. Through multiple survival analysis (Cox proportional hazards regression model), we also identified that HOXB7 expression can be used as an independent prognostic 
indicator for HNSCC. Therefore, it unbiasedly characterized that HOXB7 can be independently used as a prognostic biomarker of HNSCC.

More and more evidences show that HOXB7 is crucial to promote cell proliferation and migration to participate in tumorigenesis and inhibit cell apoptosis $[8,9,14,21]$. Consistent with this, our findings in vitro loss of function test results shows that HOXB7 down-regulated inhibit the proliferation, migration and invasion and induce apoptosis of HNSCC cells. These findings were further confirmed by facts such as decreased ability of forming tumor spheres and xenograft tumour growth after HOXB7 knowdown and positive correlation between HOXB7 expression and cervical node metastasis in our patient cohort.

Previous studies had shown that HOXB7 overexpression in hepatocellular carcinoma boosting c-Myc and Slug transportation and activated MAPK-AKT pathway to facilitate stem-like properties and EMT process in hepatoma cells, which finally malignant progression [25]. In glioma, HOXB7 facilitated the invasion and migration of tumor cells by activating the Wnt signaling, while obviously related to lymph node metastasis or distant metastasis [26]. In lung cancer, HOXB7 overexpression increases several iPSC markers and sustains the stemness of stem cell subpopulation by modulation of LIN28B [27].

Complementary, our findings investigated that several downstream targets of HOXB7, which responsible for tumorigenesis, metastasis and chemoresistance, like Snail, CD44, Vimentin and CD133, Bmi-1 clearly downregulated when HOXB7 silencing $[26,27,28,30]$. However, detailed regulatory targets of HOXB7 during HNSCC initiation and progression is still needed. In Pharmacology, HOXB7 acts as an ER co-factor, regulating the role of numerous ER targets including HER2 in tamoxifen-resistant breast cancer [29]. In this study, the CMAP analysis was performed to predicate three small molecule drugs that could potentially be targeted as a therapeutic drug HOXB7, further suggesting that HOXB7 could be a novel therapeutic target in HNSCC. Of note, our data from genetic depletion of HOXB7 and other reports from genetic inhibition of HOXB7 support the notion that HOXB7 might be a novel and viable target, which can be therapeutically manipulated to treat cancer. In conclusion, combined with cell assay in vitro and bioinformatics results extremely support the idea that HOXB7 is an newly oncogene and therapeutic target in HNSCC. Our newly coverage together with previous findings above point out that not only HOXB7 serves as a novel diagnostic and prognostic cancer biomarker, but also shows a tremendous potential as a therapeutic target.

In conclusion, this approach serves as a framework that HOXB7 is aberrantly elevated in a large cohort of HNSCC and its overexpression significantly associated with tumor aggressiveness and unfavorable survival. Broadly, RNAi-medicated HOXB7 silencing impaired tumor malignant phenotype in HNSCC. These results nominate HOXB7 may act as a master cancerous TF in HNSCC, but to further explore this, more work on finding out the exactly HOXB7 regulatory network need be down in the future as well as novel inhibitors targeting HOXB7.

\section{Declarations}

\section{Ethics approval and consent to participate}


Our whole study protocol was reviewed and approved by the Research Ethic Committee of Nanjing Medical University. Written informed consent was obtained from all subjects in our study.

\section{Consent for publication}

Not applicable

\section{Availability of data and materials}

All data generated or analyzed during this study are included in this published article and its additional files. All original data are available upon request.

\section{Competing interests}

The authors declare that they have no competing interests.

\section{Funding}

This work is financially supported, in whole or in part, by National Natural Science Foundation of China (81602378), Key Research Program in Jiangsu Province-Social Developmental Project (BE2020706), A Project Funded by the Priority Academic Program Development of Jiangsu Higher Education Institutions (2018-87).

\section{Authors' contributions}

$\mathrm{XW}$ and $\mathrm{JL}$ performed the experimental study, data collection, bioinformatic analysis and manuscript writing. TY and XK carried out some animal experiments. $X L$ and $Y Z$ performed histological and statistical analyses. JY performed surgeries and follow-up. ZL conceived and supervised the whole project. All authors have read and approved the final manuscript.

\section{Acknowledgements}

We thank Prof. Wantao Chen for his kind gift of cell lines.

\section{References}

1. Morimoto H, Yano T, Yoda Y, et al. Clinical impact of surveil-lance for head and neck cancer in patients with esophageal squamous cell carcinoma. World J Gastroenterol. 2017;23(6):1051-8.

2. Rebecca L, Siegel KD, Miller A, Jemal. Cancer statistics,2017. CA Cancer J Clin. 2020 Jan;70(1):7-30.

3. Johnson DE, Burtness B, Leemans CR, Lui VWY, Bauman JE, Grandis JR. Head and neck squamous cell carcinoma. Nat Rev Dis Primers. 2020 Nov;26(1):92. 6(.

4. William M, Lydiatt SG, Patel BO'Sullivan MS, Brandwein JA, Ridge, Jocelyn C, Migliacci, Ashley M, Loomis, Jatin P, Shah. Head and Neck cancers-major changes in the American Joint Committee on cancer eighth edition cancer staging manual. CA Cancer J Clin. 2017;67(2):122-37. 
5. Samuel S, Naora H. Homeobox gene expression in cancer: Insights from developmental regulation and deregulation. Eur J Cancer. 2005;41:2428-37.

6. Shah N, Sukumar S. The Hox genes and their roles in oncogenesis. Nat Rev Cancer. 2010;10:361-71.

7. De Souza Setubal Destro MF, Bitu CC, Zecchin KG, Graner E, Lopes MA, Kowalski LP, et al. Overexpression of HOXB7 homeobox gene in oral cancer induces cellular proliferation and is associated with poor prognosis. Int J Oncol. 2010;36:141-9.

8. Chen H, Lee JS, Liang X, Zhang H, Zhu T, Zhang Z, et al. HOXB7 inhibits transgenic HER-2/neuinduced mouse mammary tumor onset but promotes progression and lung metastasis. Cancer Res. 2008;68:3637-44.

9. Wu X, Chen H, Parker B, Rubin E, Zhu T, Lee JS, et al. HOXB7, a homeodomain protein, is overexpressed in breast cancer and confers epithelial-mesenchymal transition. Cancer Res. 2006;66:9527-34.

10. Tu W, Zhu X, Han Y, Wen Y, Qiu G, Zhou C. Overexpression of HOXB7 is associated with a poor prognosis in patients with gastric cancer. Oncol Lett. 2015;10:2967-73.

11. Lijun Cai Z, Wang $H$, Zheng $L, X u$. The let-7c/HoxB7 axis regulates the cell proliferation, migration and apoptosis in hepatocellular carcinoma. Anticancer Drugs. 2020 Jan;31(1):6-18.

12. Cheng W, Shi X, Lin M, Yao Q, Ma J, Li J. LncRNA MAGI2-AS3 Overexpression Sensitizes Esophageal Cancer Cells to Irradiation Through Down-Regulation of HOXB7 via EZH2. Front Cell Dev Biol. 2020;8:552822.

13. Li H, Shen LY, Yan WP, Dong B, Kang XZ, Dai L, et al. Deregulated HOXB7 Expression Predicts Poor Prognosis of Patients with Esophageal Squamous Cell Carcinoma and Regulates Cancer Cell Proliferation In Vitro and In Vivo. PLoS ONE. 2015;10:e0130551.

14. Nguyen Kovochich A, Arensman M, Lay AR, Rao NP, Donahue T, Li X, et al. HOXB7 promotes invasion and predicts survival in pancreatic adenocarcinoma. Cancer. 2013;119:529-39.

15. Makiko Tsuboi K, Taniuchi T, Shimizu M, Saito, Toshiji Saibara. The transcription factor HOXB7 regulates ERK kinase activity and thereby stimulates the motility and invasiveness of pancreatic cancer cells. J Biol Chem. 2017;292(43):17681-702.

16. Zhangxing Song Z, Liao Y, Cui C. Yang. The relationship between homeobox B7 expression and the clinical characteristics of patient with prostate cancer. J Cell Biochem. 2019;120(4):6395-401.

17. Jin K, Park S, Teo WW, Korangath P, Cho SS, Yoshida T, Győrffy B, Goswami CP, Nakshatri H, Cruz LA, Zhou W, Ji H, Su Y, Ekram M, Wu Z, Zhu T, Polyak K, Sukumar S. HOXB7 Is an ERaCofactor in the Activation of HER2 and Multiple ER Target Genes Leading to Endocrine Resistance. Cancer Discov. 2015;5(9):944-59.

18. Yuan W, Zhang X, Xu Yu, Li S, Hu Y. Shiyong Wu. Role of HOXB7 in regulation of progression and metastasis of human lung adenocarcinoma. Mol Carcinog. 2014;53(1):49-57.

19. Xie X, Zhang Shui-shen, Wen J, Yang H, Luo Kong-jia, Yang Fu. Yi Hu, Jian-hua Fu. Prognostic value of HOXB7 mRNA expression in human oesophageal squamous cell cancer. Biomarkers. 2013;18(4):297-303. 
20. Zhang E-b, Yin D-d, Sun M, Kong R, Liu X-h, You L-h, Han L, Xia R, Wang K-m, Yang J-s, De W, Shu Y-q. $Z-x$ Wang. P53-regulated long non-coding RNA TUG1 affects cell proliferation in human non-small cell lung cancer, partly through epigenetically regulating HOXB7 expression. Cell Death Dis. 2014;5(5):e1243.

21. Joo MK, Park JJ, Yoo HS, Lee BJ, Chun HJ, Lee SW, et al. The roles of HOXB7 in promoting migration, invasion, and antiapoptosis in gastric cancer. J Gastroenterol Hepatol. 2016;31:1717-26.

22. Cheng W, Shi X, Lin M, Yao Q, Ma J, Li J. LncRNA MAGI2-AS3 Overexpression Sensitizes Esophageal Cancer Cells to Irradiation Through Down-Regulation of HOXB7 via EZH2. Front Cell Dev Biol. 2020 Nov 24;8:552822.

23. Gavrielatou N, Doumas S, Economopoulou P, Foukas PG, Psyrri A. Biomarkers for immunotherapy response in head and neck cancer. Cancer Treat Rev. 2020 Mar;84:101977.

24. Johnson DE, Burtness B, Leemans CR, Lui VWY, Bauman JE, Grandis JR. Head and neck squamous cell carcinoma.

25. Nat Rev Dis Primers. 2020 Nov 26;6(1):92.

26. Huan HB, Yang DP, Wen XD, Chen XJ, Zhang L, Wu LL, Bie P, Xia F. HOXB7 accelerates the malignant progression of hepatocellular carcinoma by promoting stemness and epithelial-mesenchymal transition. J Exp Clin Cancer Res. 2017 Jun;24(1):86. 36(.

27. Huo 1 X-Y, Zhang X-Y, Yuan F, Zhao X-Y, You B-A. HOXB7 promotes proliferation and metastasis of glioma by regulating the Wnt/ $\beta$-catenin pathway. Eur Rev Med Pharmacol Sci. 2019 Mar;23(6):247685.

28. Simona Monterisi PL, Riso K, Russo G, Bertalot M, Vecchi. Giuseppe Testa, Pier Paolo Di Fiore, Fabrizio Bianchi. HOXB7 overexpression in lung cancer is a hallmark of acquired stem-like phenotype. Oncogene. 2018 Jun;37(26):3575-88.

29. Monterisi S, Lo Riso P, Russo K, Bertalot G, Vecchi M, Testa G, Di Fiore PP, Bianchi F. HOXB7 overexpression in lung cancer is a hallmark of acquired stem-like phenotype. Oncogene. 2018 Jun;37(26):3575-88.

30. Jin K, Park S, Teo WW, Korangath P, Cho SS, Yoshida T, Balázs Győrffy, Chirayu Pankaj Goswami, Harikrishna Nakshatri, Leigh-Ann Cruz, Weiqiang Zhou, Hongkai Ji, Ying Su, Muhammad Ekram, Wu Z, Zhu T, Kornelia Polyak, Saraswati Sukumar. HOXB7 Is an ERa Cofactor in the Activation of HER2 and Multiple ER Target Genes Leading to Endocrine Resistance. Cancer Discov. 2015 Sep;5(9):944 59.

31. Liping Wu Y, Liu C, Guo. Yuan Shao. LncRNA OIP5-AS1 promotes the malignancy of pancreatic ductal adenocarcinoma via regulating miR-429/FOXD1/ERK pathway. Cancer Cell Int. 2020;20:296.

\section{Figures}


A

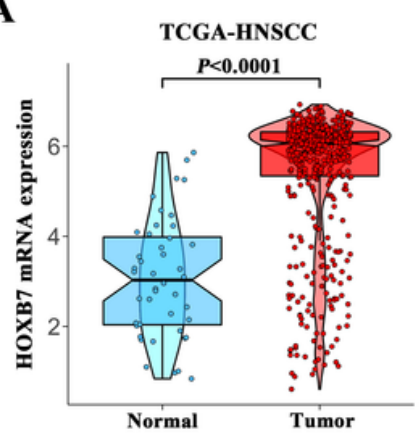

E

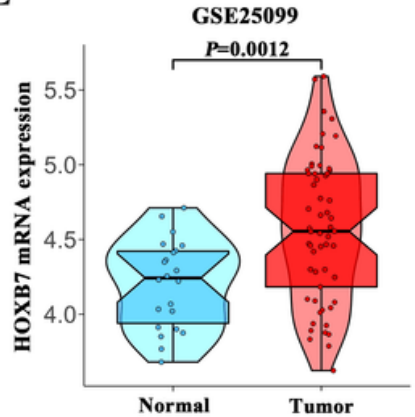

B

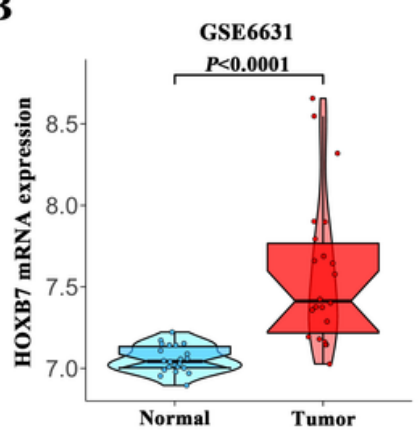

F

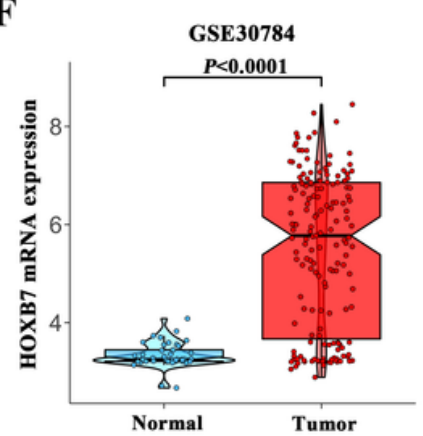

C

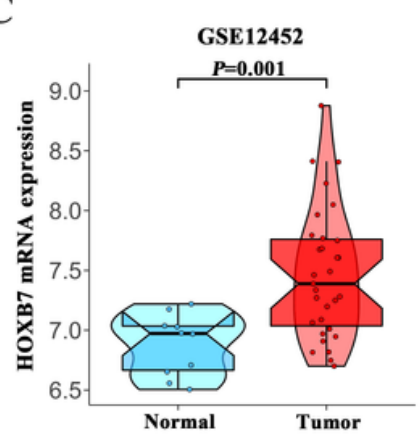

G

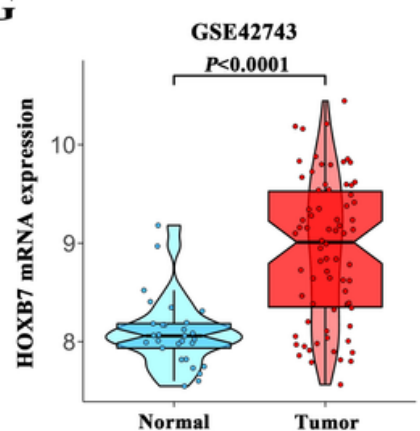

D

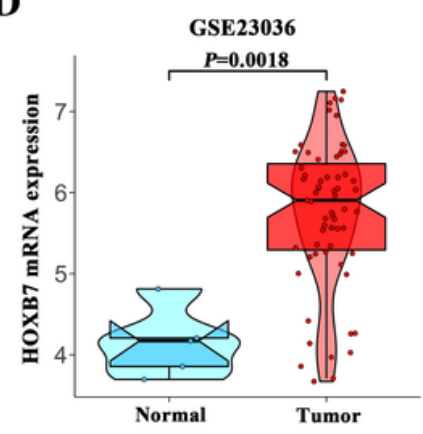

H

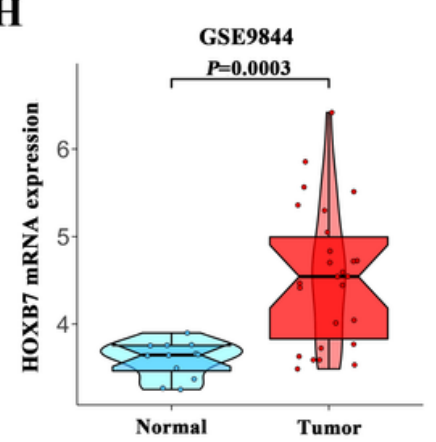

Figure 1

Overexpression of HOXB7 mRNA in HNSCC datasets. A-H: The mRNA levels of HOXB7 (normalized and log2-transformed) were compared between HNSCC samples and normal counterparts in TCGA-HNSC (A), GSE6631(B), GSE12452(C), GSE23036(D), GSE25099(E), GSE30784(F), GSE42743(G) and GSE9844(H) datasets. The original data were retrieved from GEO database and TCGA, then plotted using Graphpad Prism 6.0 software. HNSCC, head neck squamous cell carcinoma; HOXB7, Homeobox B7. 
A

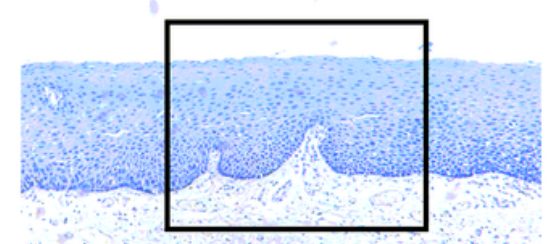

B

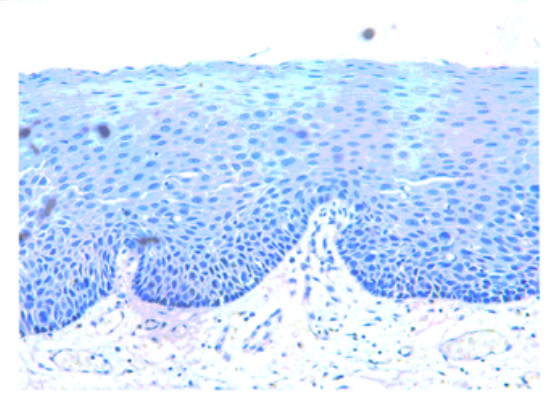

C

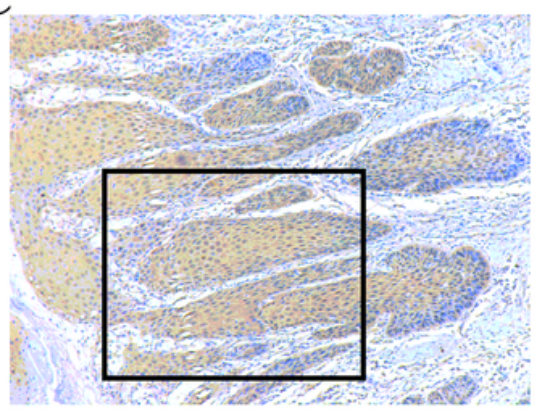

D

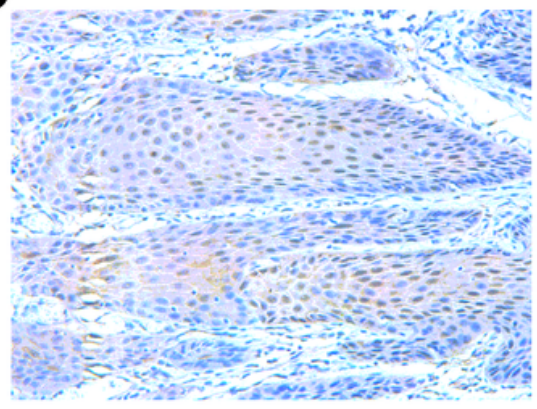

E

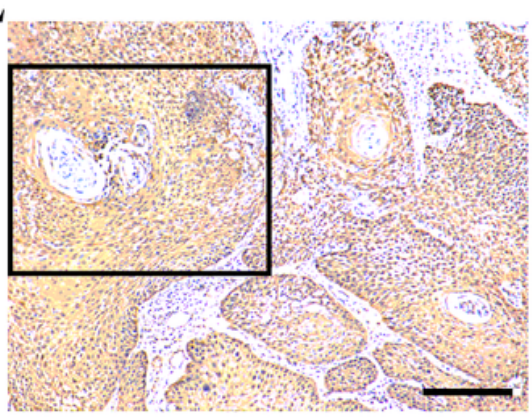

F

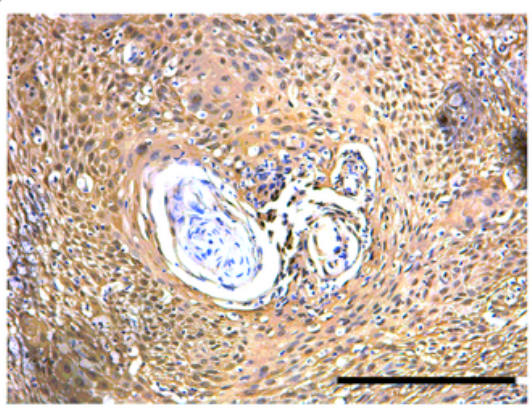

G

HOXB7

Actin

DAPI

Merge

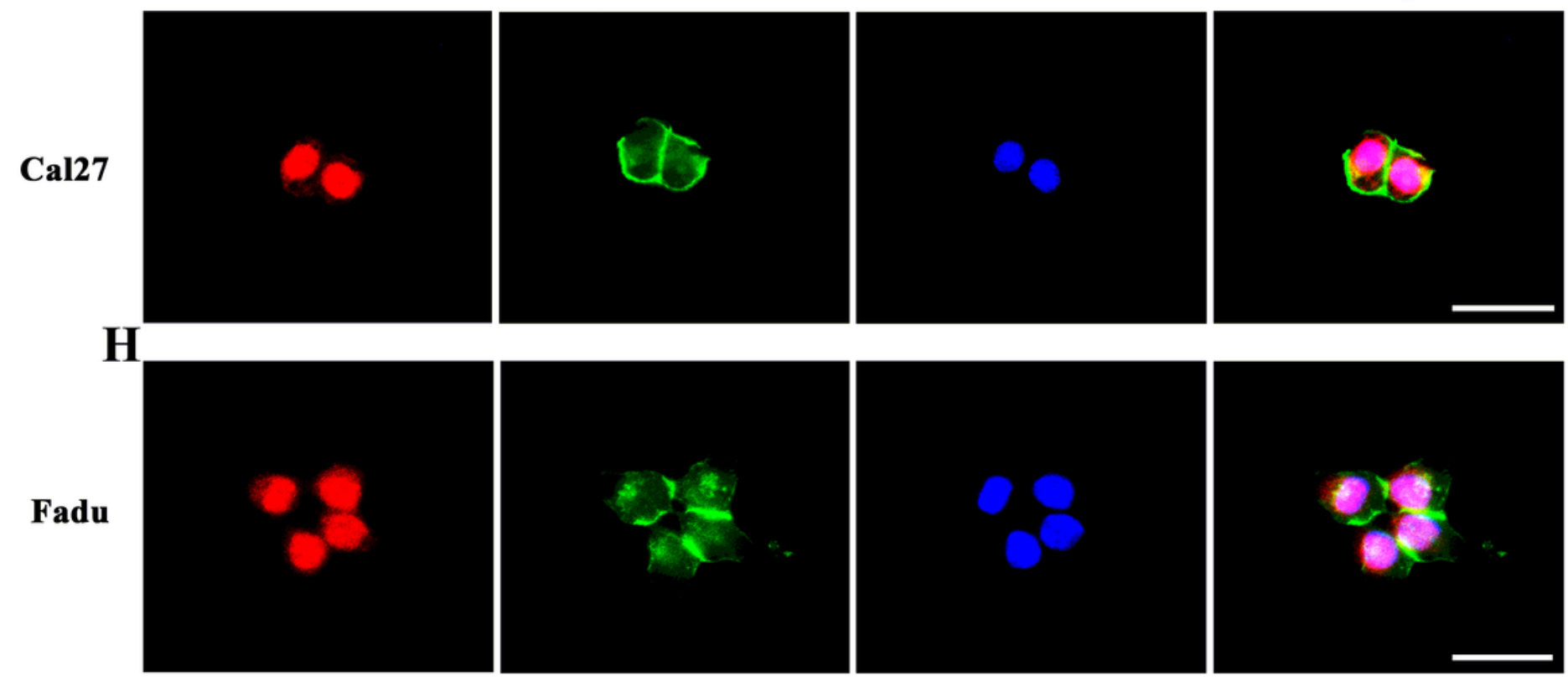

\section{Figure 2}

Nuclear location HOXB7 enriched in human HNSCC samples. A, B: Representative negative staining of HOXB7 in normal oral epithelial $(100 \times A, 200 \times B)$; C, D: Representative low expression of HOXB7 in primary human HNSCC sample $(100 \times C, 200 \times D)$; E, F: Representative high expression of HOXB7 in primary human HNSCC sample (100× E, 200× F). G, H: HOXB7 cytoplasmic and nuclear localization in HNSCC cell lines. HOXB7 was predominantly identifified in nucleus and rarely in cytoplasm. Nuclei are counterstained with DAPI. Scale bar: $100 \mu \mathrm{m}$. 
A

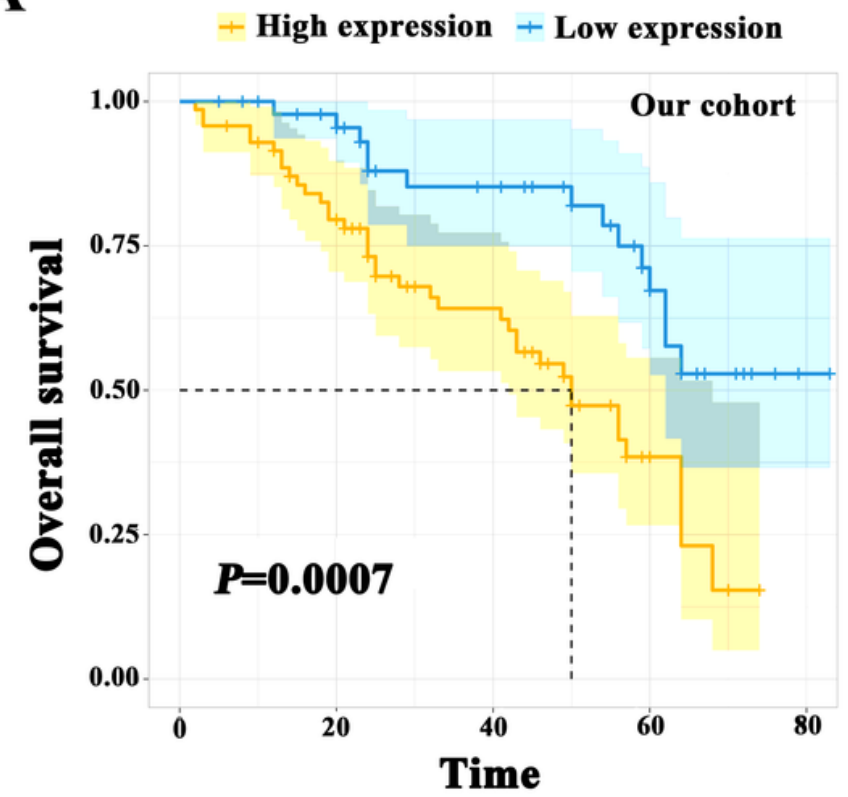

C

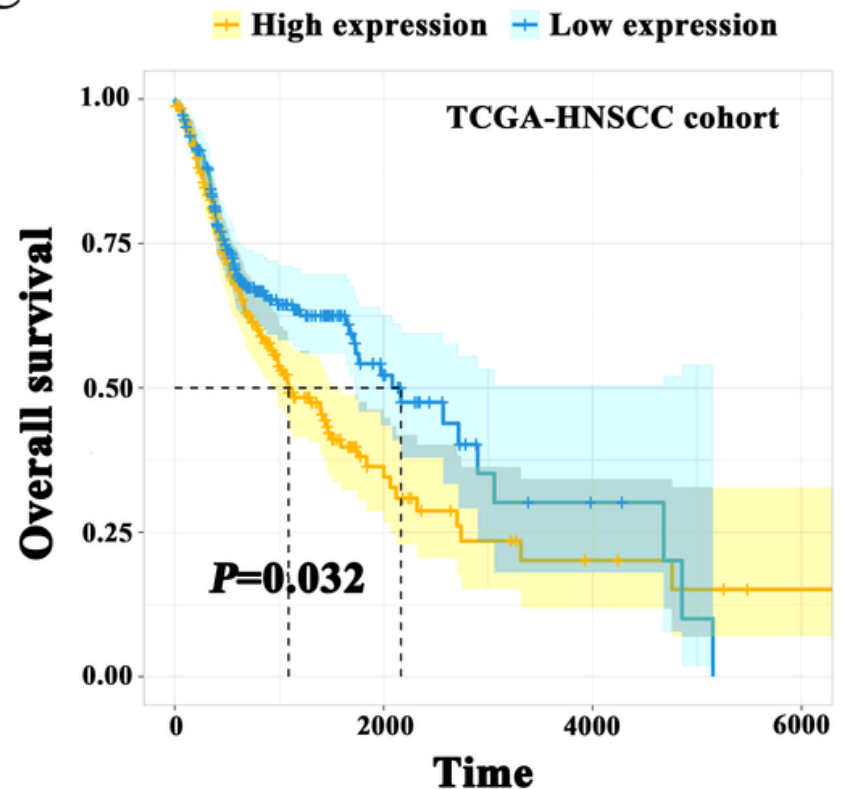

B

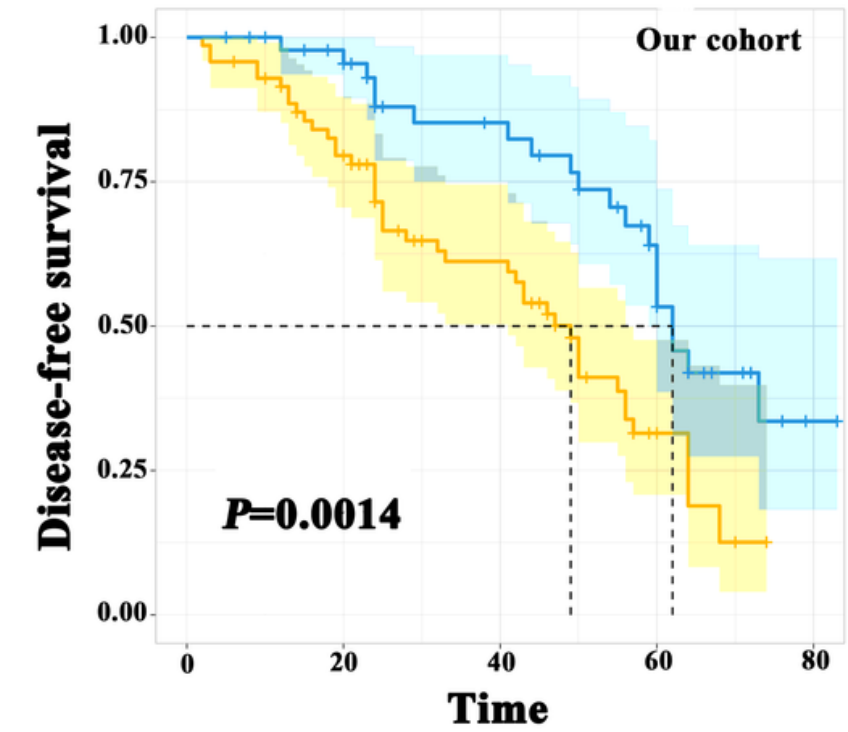

D

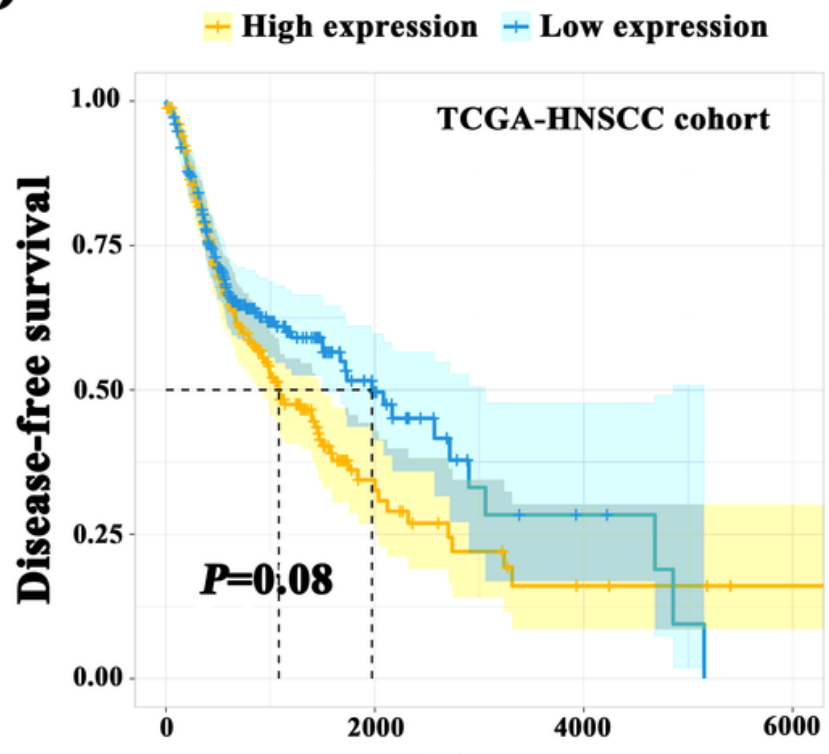

Time

Figure 3

High HOXB7 expression positively associates with reduced overall survival rates in HNSCC patients. A, B: Overall survival (A) and disease-free survival (B) analyses of HNSCC patients with high or low expression of FOXD1 were estimated by IHC; C, D: Overall survival (C) and disease-free survival (D) plot of FOXD1 in TCGA-HNSC patients downloaded from cBioPortal(https://www.cbioportal.org/). Kaplan-Meier method with median cutoff and compared with Log-rank t-test. 
A

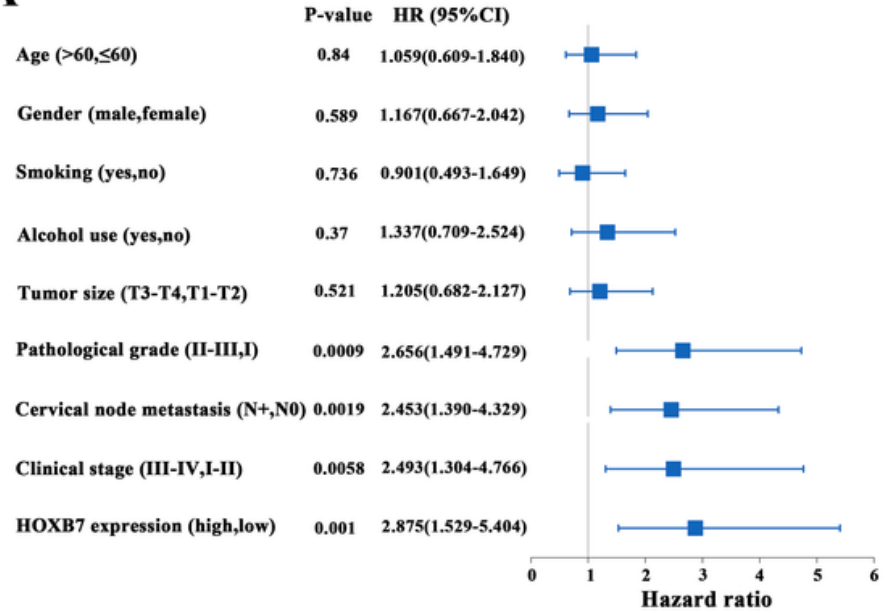

B

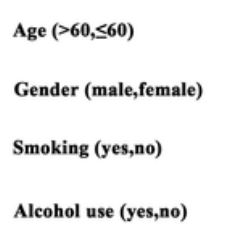

Tumor size (T3-T4,T1-T2)

Pathological grade (II-III,I)

Cervical node metastasis $(\mathrm{N}+, \mathrm{N} 0) 0.6666 \quad 1.228(0.483-3.121)$

Clinical stage (III-IV,I-II)

HOXB7 expression (high,low)

P-value HR (95\%CI)

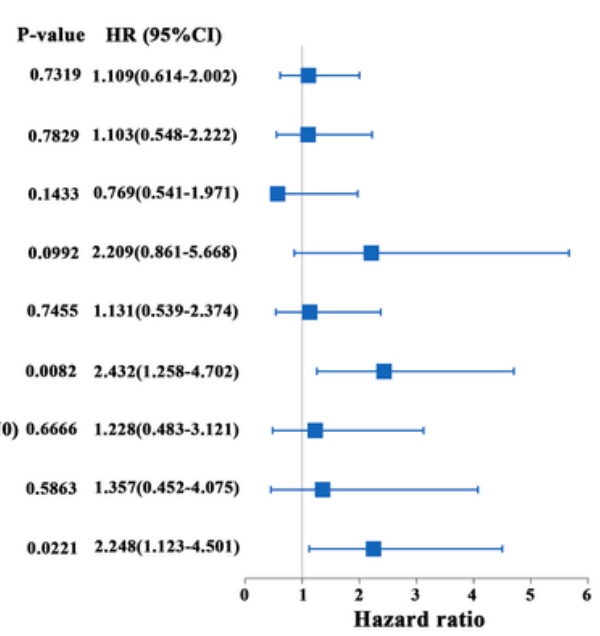

\section{Figure 4}

Univariate and multivariate survival analyses (proportional hazards method) for patients with primary HNSCC. A: The univariate survival analysis revealed that HOXB7 expression, pathological grade, cervical nodal metastasis and clinical stage were significantly associated with overall survival; B: Multivariate survival analysis indicated HOXB7 abundance and pathological grade were found to be an independent prognostic marker for the overall survival of patients with HNSCC. Cox proportional hazards regression model. 


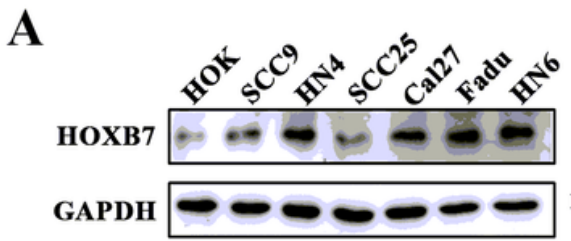

B

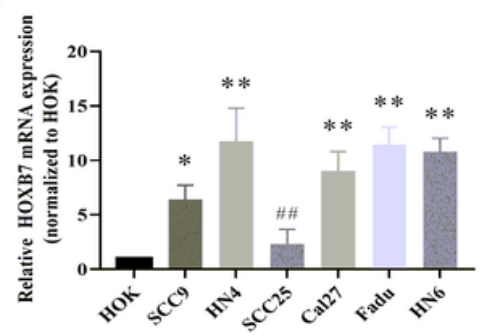

E

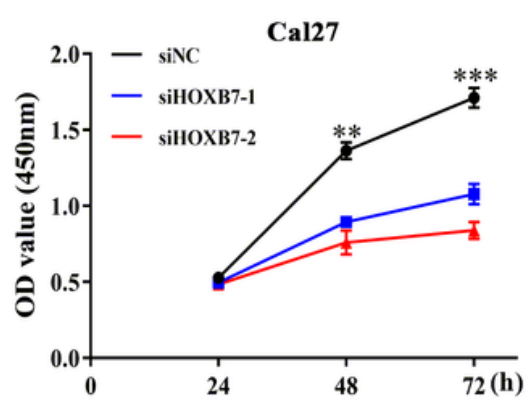

I

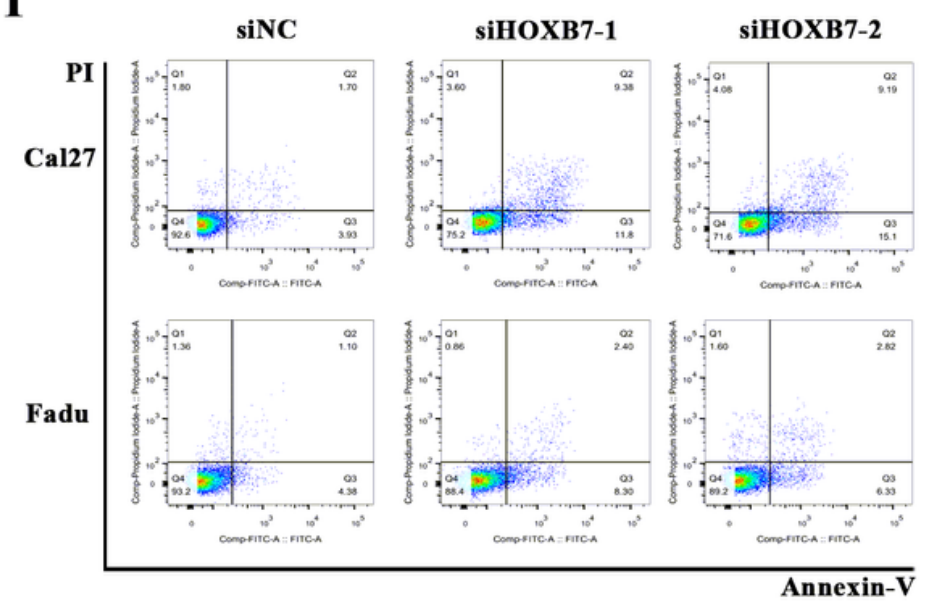

C
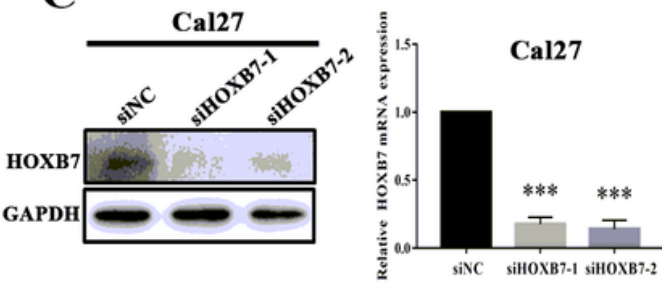

D
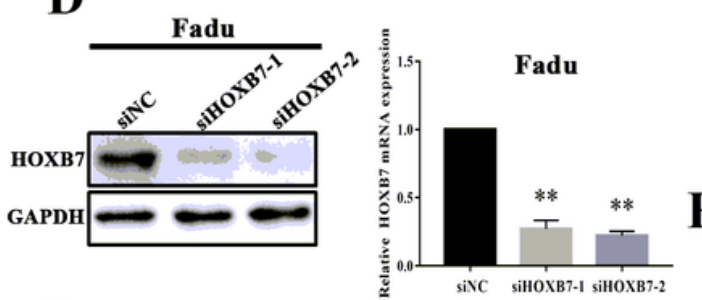

F

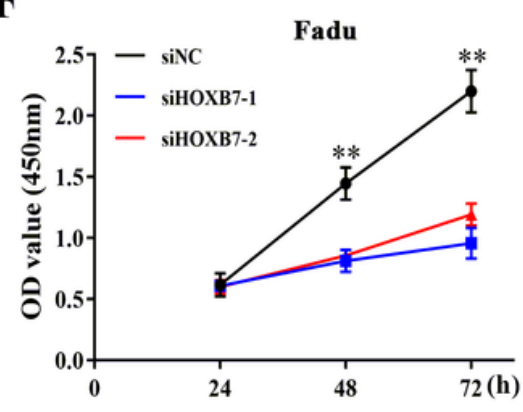

G
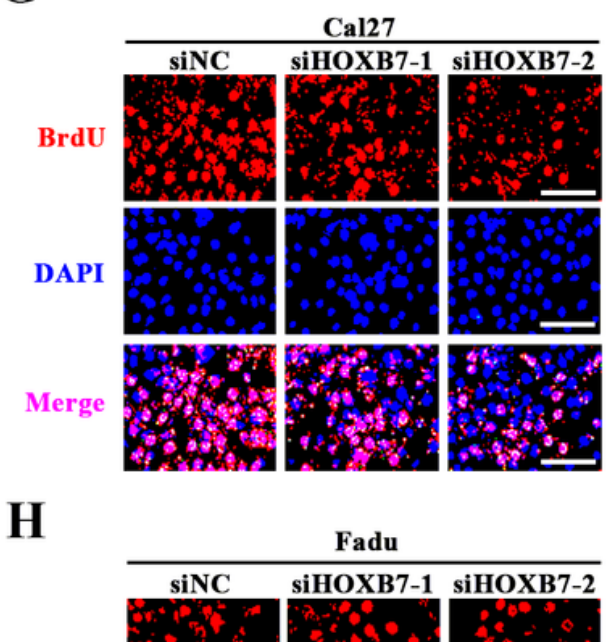

BrdU

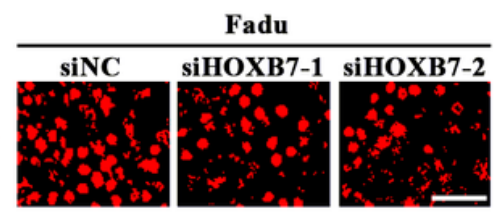

DAPI
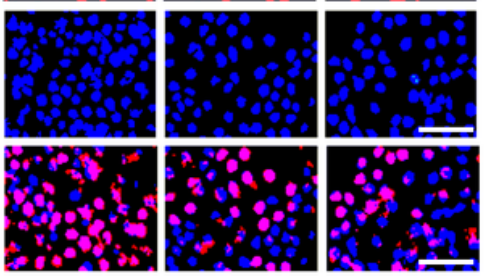

Merge

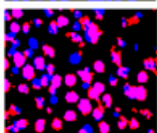

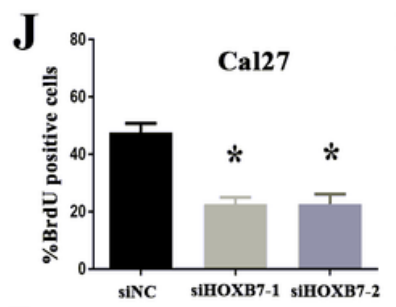

L
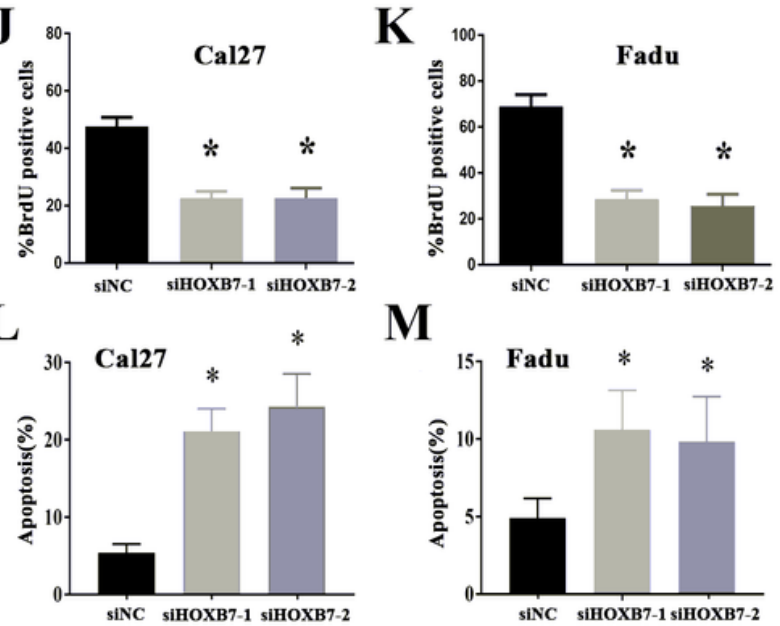

M

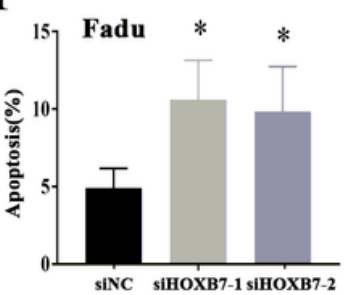

Figure 5

HOXB7 depletion impairs proliferation and triggers apoptosis in HNSCC cells in vitro. A, B: Endogenous HOXB7 protein and mRNA expression were measured in a panel of HNSCC cell lines as compared to normal human oral keratinocytes (HOK); C, D: Endogenous HOXB7 protein and mRNA were efficiently silenced by two siRNAs (siHOXB7-1, siHOXB7-2) in FADU and Cal27 cells. Non-targeting siRNA was utilized as negative control (siNC); E-H, J, K: Cell proliferation was remarkably suppressed when endogenous HOXB7 was silenced as measured by CCK8 and BrdU assays; I-M: Increased percentages of cell undergoing apoptosis were evident following HOXB7 knockdown as assayed by Annexin V-PI staining. Scale bar: $100 \mu \mathrm{m}$. Data shown from three independent experiments, ${ }^{*} \mathrm{P}<.05,{ }^{\star \star} \mathrm{P}<.01$, Student's t-test. 


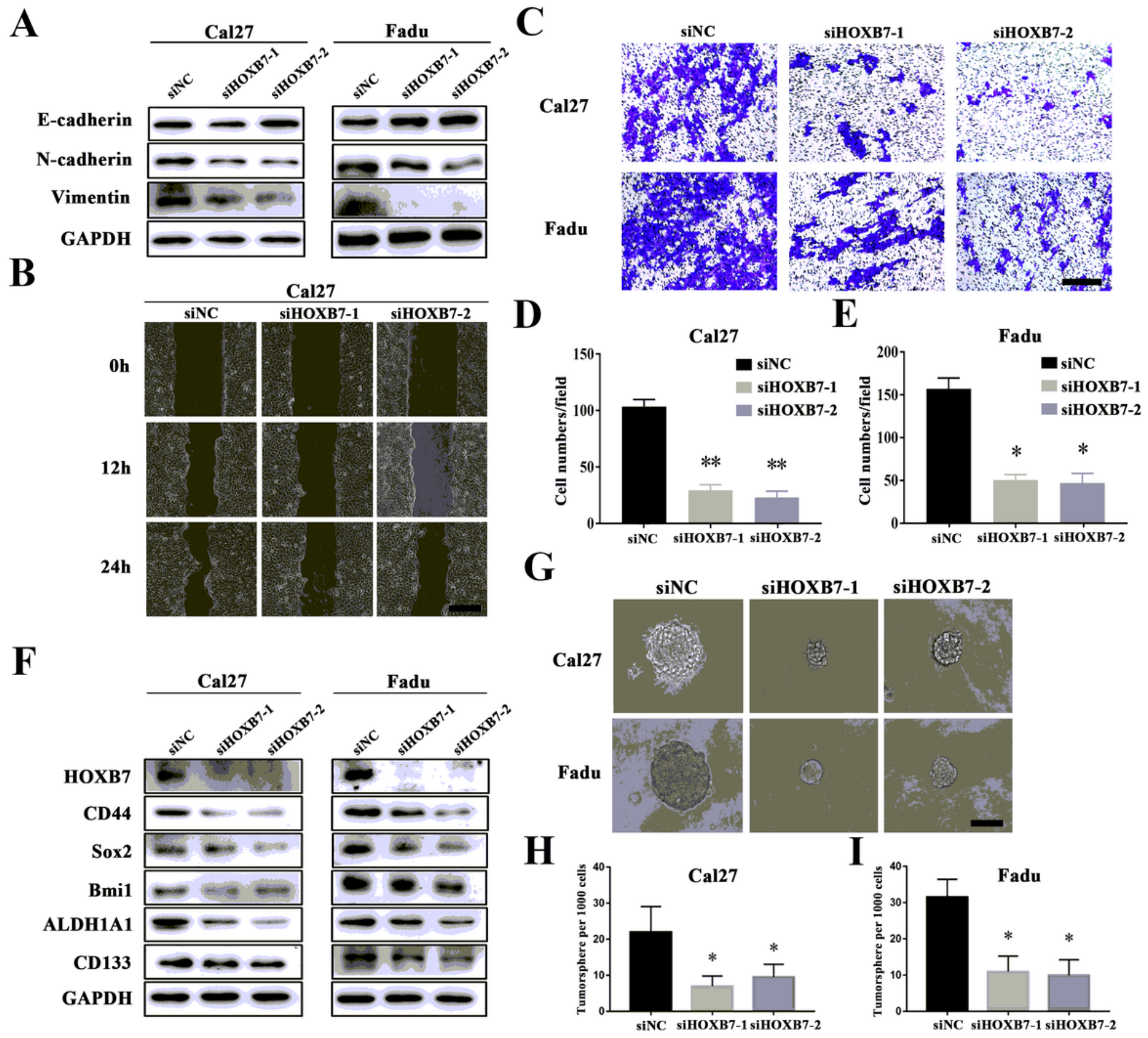

Figure 6

HOXB7 knockdown inhibited migration and invasion and impaired CSCs maintenance in HNSCC. A, The abundance of EMT-related markers E-cadherin, N-cadherin and vimentin were measured by western blot (WB) in the Cal27 and Fadu cells following HOXB7 knockdown; B-E, The cell motilities and invasion were remarkably diminished after HOXB7 knockdown as gauged by wound healing (B) and transwell-invasion assay (C, D, E). Measurements of wound healing was performed at 12 and $24 \mathrm{~h}$ after cell scratching while measurements of transwell assays were done at $12 \mathrm{~h}$ after cell seeding; $\mathrm{G}$, The capability of tumorsphere formation was markedly reduced in siHOXB7-transfected cells relative to control cells. H, I, Quantification of primary and secondary tumorspheres culture were shown; $F$, The abundances of CSCs makers were measured in cells after HOXB7 inhibition. Scale bar $=50 \mu \mathrm{m}$. Data are mean \pm SD; the Student t test. 
CSCs, cancer stem cells; HNSCC, head-neck squamous cell carcinoma; SD, standard deviation. ${ }^{*}<<0.05$; $\star * \mathrm{P}<0.01$.

A
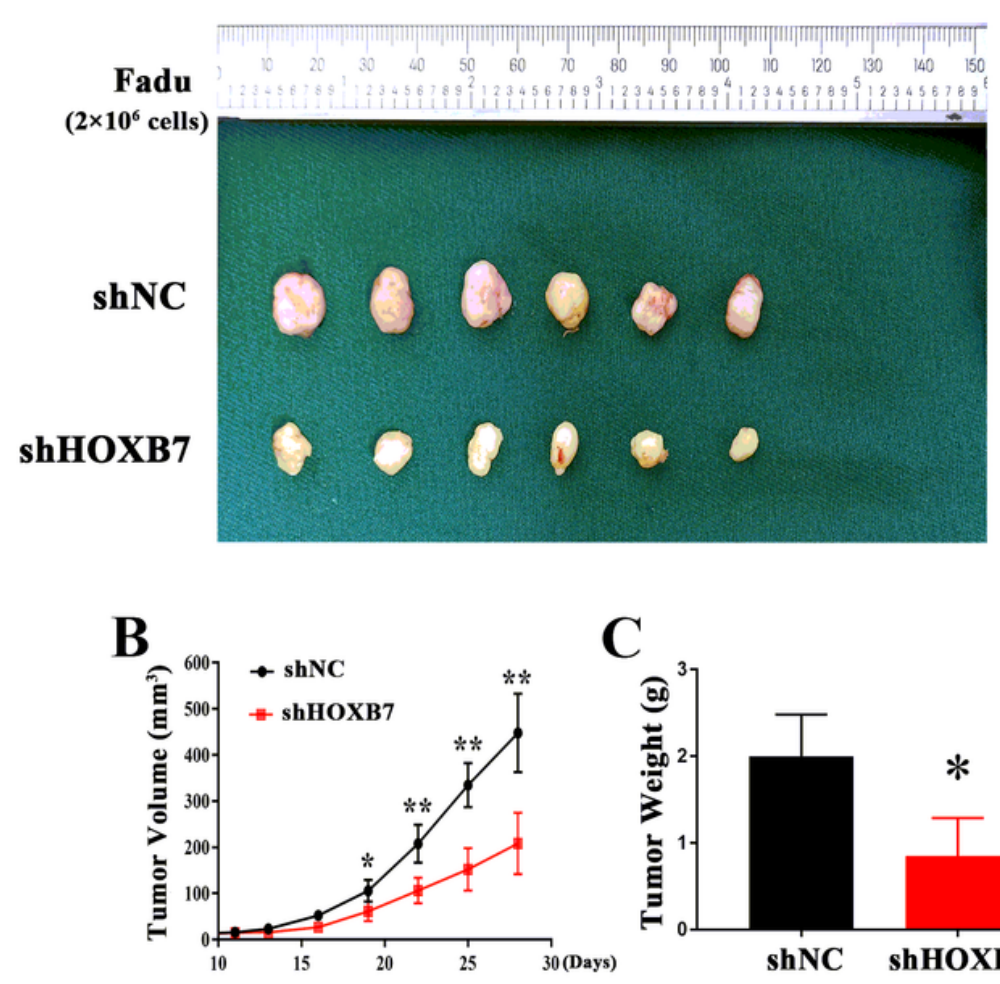

D

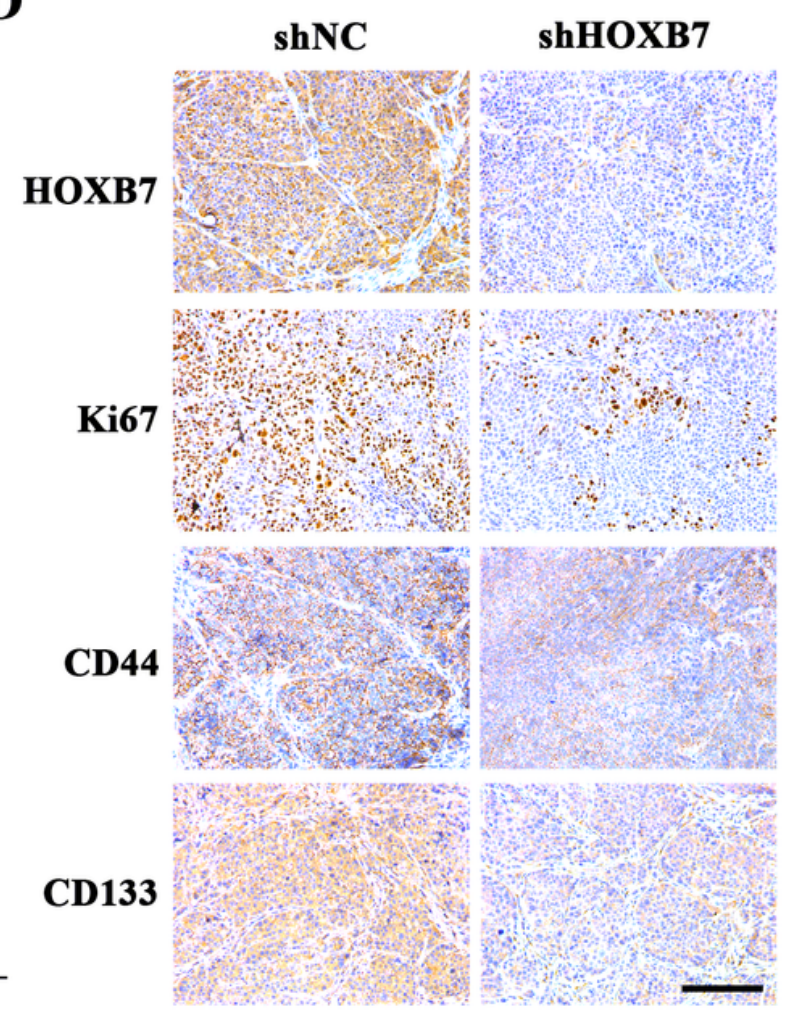

\section{Figure 7}

HOXB7 depletion impaired tumor growth in a HNSCC xenograft model. A-B, Tumour volume was monitored in xenograft samples derived from FADU cells with stable HOXB7 knockdown or controls; $C$, Final weight of tumour masses harvested from derived from FADU cells with stable HOXB7 knockdown or controls was compared. D, The marker indicative of HOXB7 knockdown HOXB7 and proliferative marker Ki67 and CSCs markers CD44 and CD133 were determined by immunohistochemical staining in xenograft samples derived from FaDu cells with stable HOXB7 knockdown or controls. Scale bar: $100 \mathrm{Im}$. Representative images are shown. ${ }^{*} \mathrm{P}<.05,{ }^{\star} \mathrm{P}<.01$, Student's t-test. 
A

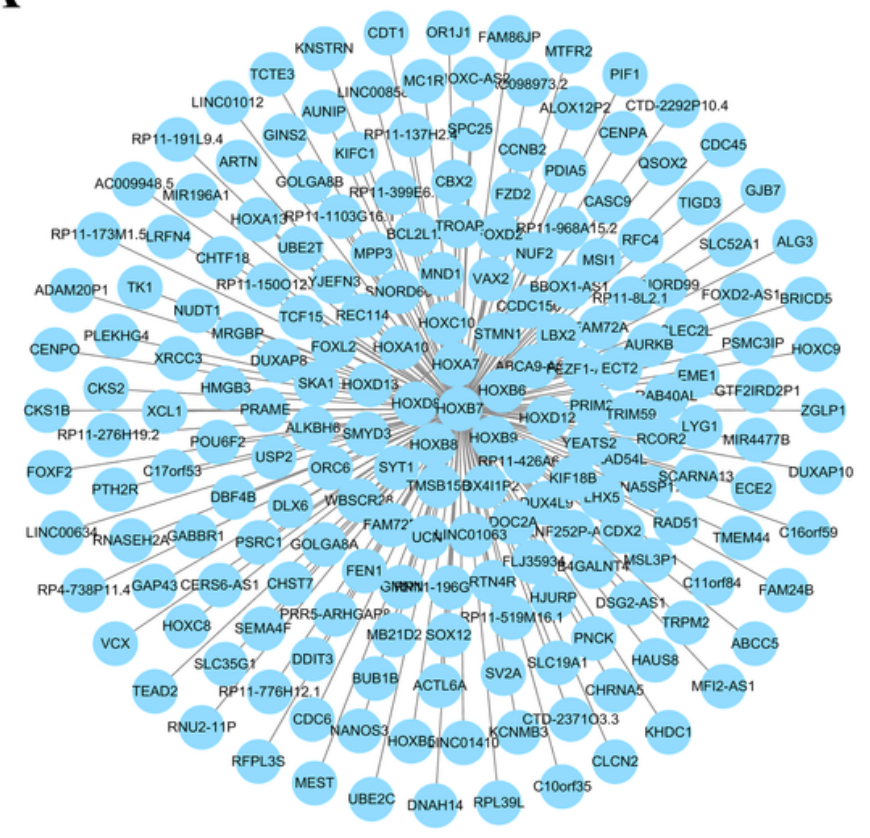

B

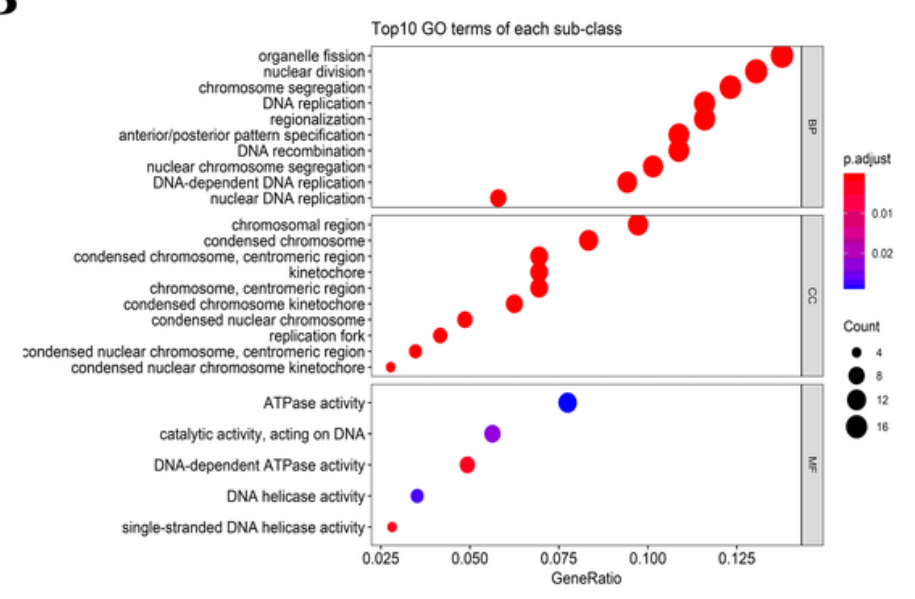

C

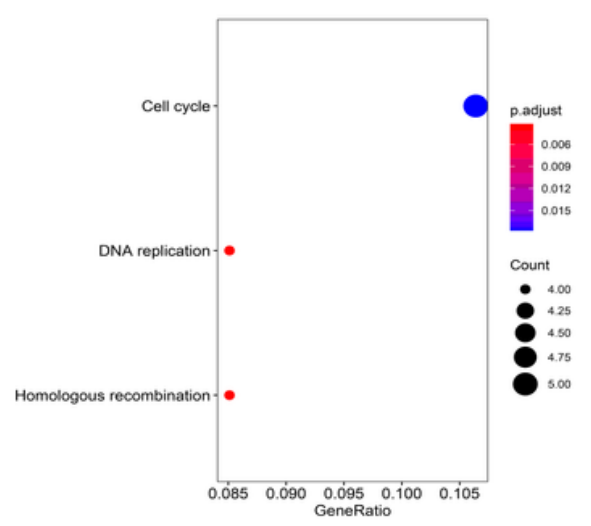

Figure 8

Therapeutic potential analysis on HOXB7 in HNSCC. A, Screen and identify HOXB7-related genes with a $\mathrm{P}$ $<0.05$ and $|r|>0.3$ were identified as HOXB7-related genes by using the Pearson correlation coefficient (r); $\mathrm{B}-\mathrm{C}$, The biological functions of these HOXB7 co-expression genes were comprehensively detected by GO enrichment and kyoto encyclopedia of genes and genomes (KEGG) pathway analysis.

\section{Supplementary Files}

This is a list of supplementary files associated with this preprint. Click to download.

- SupplementaryFigure1.tif 